\title{
Aortopulmonary fistula in pseudoaneurysm after ascending aortic surgery
}

Tadashi Kitamura, MD, Noboru Motomura, MD, Toshiya Ohtsuka, MD, Ko Shibata, MD, Hiroo Takayama, MD, Yutaka Kotsuka, MD, and Shinichi Takamoto, MD, Tokyo, Japan

$\mathrm{P}$ seudoaneurysm after graft replacement of the ascending aorta is often fatal, although rupture into the pulmonary artery is extremely rare. We report an exceptional case of pseudoaneurysm of the ascending aorta with fistulization into the pulmonary artery.

\section{Clinical Summary}

A 52-year-old man had undergone, in another hospital, a graft replacement of the ascending aorta and coronary bypass grafting (saphenous vein to the right coronary artery) for acute type A aortic dissection. Gelatin-resorcinol-formaldehyde tissue glue had been used in reinforcement of the dissected aorta in both ends.

Nine months after that operation, the patient had dyspnea and mild fever. Computed tomographic (CT) scan showed pseudoaneurysm of the ascending aorta, and he was referred to us. Physical findings included a continuous murmur maximal at the second right intercostal space. Chest radiography showed mild cardiomegaly and enhancement of the right pulmonary vasculature. Transesophageal echocardiography and a new CT scan (Figure 1) revealed a pseudoaneurysm of the ascending aorta, with fistulization to the right pulmonary artery arising from the proximal anastomosis. Aortography confirmed complete occlusion of the vein graft as well as the presence of the aortopulmonary fistula. Right heart catheterization revealed right-sided pressure elevation. When the left ventricular pressure was $74 / 20 \mathrm{~mm} \mathrm{Hg}$, the right atrial pressure was $17 \mathrm{~mm} \mathrm{Hg}$, the right ventricular was $34 / 18 \mathrm{~mm} \mathrm{Hg}$, and the pulmonary arterial pressure was $35 / 16 \mathrm{~mm} \mathrm{Hg}$. An oxygen saturation step-up was observed in the pulmonary artery, with $59.8 \%$ in the main pulmonary artery and $91.8 \%$ in the right pulmonary artery. Because of the risks of repeated median sternotomy, we chose a right thoracotomy to approach the aortic root. We prepared a cryopreserved aortic valve allograft in case of infection.

The right fourth intercostal space was opened, and sternal transection and left thoracotomy were included. The femoral artery and the superior and inferior venae cavae were cannulated for cardiopulmonary bypass. An occlusion balloon was inserted into

\footnotetext{
From the Department of Cardiothoracic Surgery, Graduate School of Medicine, University of Tokyo, Tokyo, Japan.

Received for publication March 30, 2003; accepted for publication April 8, 2003.

Address for reprints: Tadashi Kitamura, MD, University of Tokyo, Cardiovascular Surgery, 7-3-1, Hongo, Bunkyo-ku, Tokyo 113-8655, Japan (Email: funcorogash@ hotmail.com).

J Thorac Cardiovasc Surg 2003;126:904-5

Copyright (C) 2003 by The American Association for Thoracic Surgery $0022-5223 / 2003 \$ 30.00+0$

doi:10.1016/S0022-5223(03)00712-8
}

the distal part of the pseudoaneurysm to reduce the degree of aortopulmonary shunting. After systemic cooling, retrograde cardioplegia, and deep hypothermic circulatory arrest, the pseu-

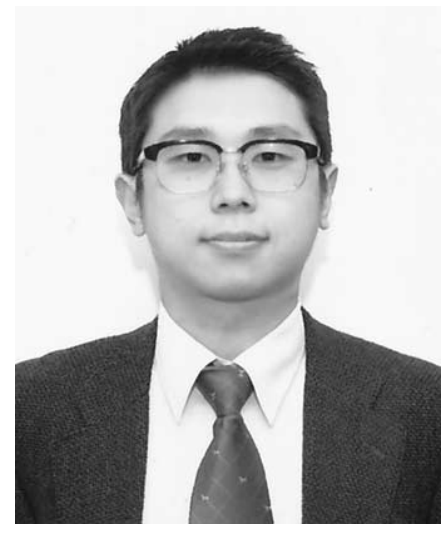

Dr Kitamura doaneurysm was incised, and dissection proceeded until the distal part of the aortic graft was crossclamped and systemic circulation was resumed. The defect of the right pulmonary artery wall was $14 \mathrm{~mm}$ in diameter; it was closed with the anterior mitral leaflet taken from the aortic allograft. We found dehiscence in the anterior part of the proximal anastomosis, blackened aortic edge, and fragile aortic wall with dissection almost reaching the annulus. There were no findings indicating infection. The left coronary ostium was resected as a button. A Dacron polyester fabric tube composite graft with a mechanical valve was inserted into the aortic root, and the left coronary button was reattached to the graft. During rewarming, the right internal thoracic artery was anastomosed to the right coronary artery (Figure 2).

The patient's postoperative course included pneumonia, which improved with antibiotics, and he was discharged 47 days after operation. Histologic examination of the media of the dissected aorta revealed a homogeneous eosinophilic substance that seemed to be composed of glue, severe calcification, and fibrous tissue with hemosiderin deposition.

\section{Discussion}

Pseudoaneurysm of the ascending aorta, which is only rarely complicated by aortopulmonary fistula, ${ }^{1,2}$ may result in death from rupture. Murmurs, echocardiography, CT scan, aortography, and right heart catheterization can potentially confirm the diagnosis of aortopulmonary fistula.

In this case we chose right thoracotomy together with sternal transection and left thoracotomy because extensive adhesion was anticipated on the basis of the CT scan. Aortic root replacement and coronary artery bypass grafting were successfully performed through this approach. The aortopulmonary fistula caused so much shunting that the oxygen saturation in the right pulmonary artery was $91.8 \%$. We used an occlusion balloon to reduce the shunt flow during cardiopulmonary bypass.

Some reports have pointed out the possible adverse effectsregarding redissection- of gelatin-resorcinol-formaldehyde glue used in the repair of type A aortic dissection. ${ }^{3,4}$ In those studies, histologic examination of the redissected aorta showed disappearance of cells or nuclei along with hemosiderin deposition. Similarly, in this case both fibrous tissue with hemosiderin deposition and severe calcification were confirmed in the redissected media. 


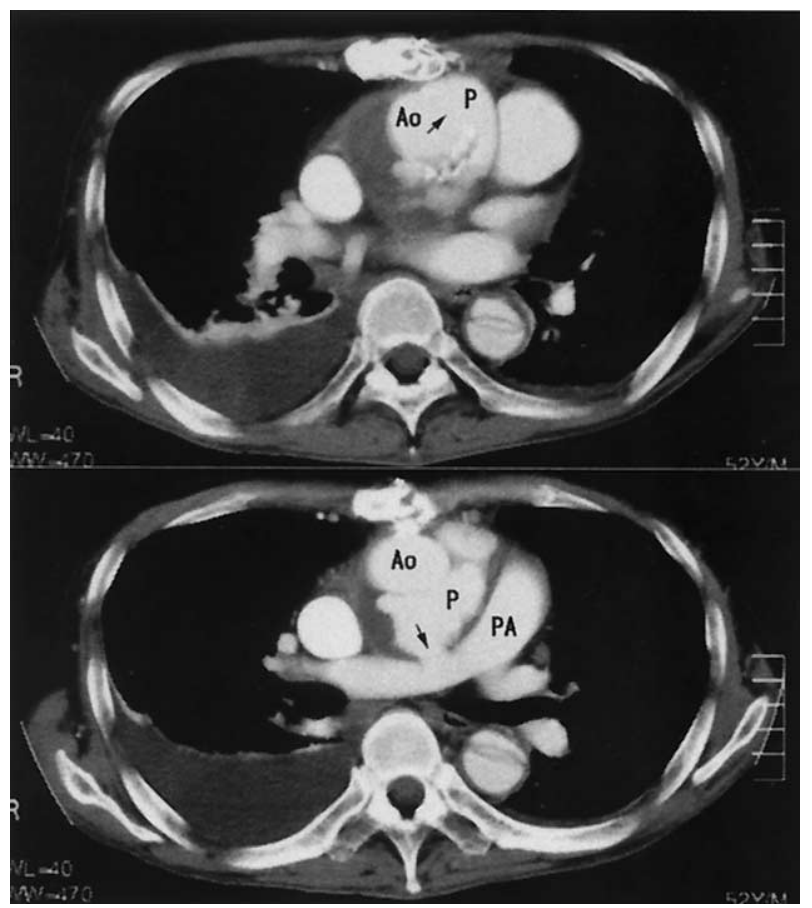

Figure 1. Preoperative CT scan. Arrows indicate dehiscence of proximal anastomosis (upper) and fistulization to pulmonary artery (PA, lower). Ao, Aorta; $P$, pseudoaneurysm.

Formaldehyde, which can leach from gelatin-resorcinol-formaldehyde glue, has a necrotic action on tissues, which may cause dehiscence and pseudoaneurysm in the anastomosis.

When a patient is seen with congestive heart failure with continuous heart murmur after ascending aortic surgery, aortopulmonary fistula should be considered. Success is facilitated by an accurate strategy for separation of the systemic and pulmonary circulation.
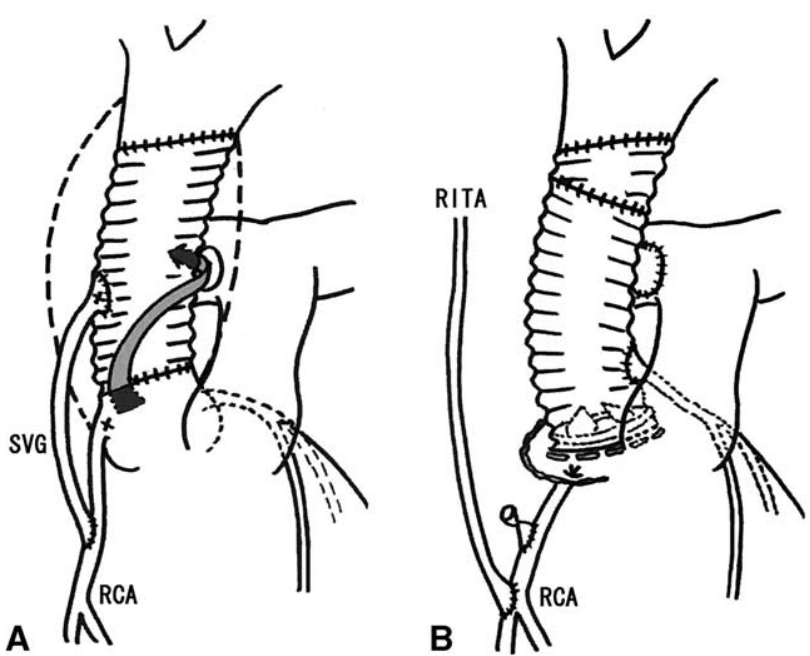

Figure 2. Schematic drawing of preoperative (A) and postoperative (B) findings. Interrupted line indicates the pseudoaneurysm; arrow shows aortopulmonary shunting. SVG, Saphenous vein graft; $R C A$, right coronary artery; RITA, right internal thoracic artery.

\section{References}

1. Chevalier P, Moncada E, Kirkorioan G, Touboul P. Acquired aortopulmonary fistula in pseudoaneurysm of the aorta six years after a Bentall operation. J Thorac Cardiovasc Surg. 1995;110:1143-4.

2. Ueno M, Imada $\mathrm{T}$, Nonaka $\mathrm{K}$, Oda $\mathrm{T}$. Aortopulmonary fistula after aortic root replacement. Ann Thorac Surg. 2002;74:590-1.

3. Bingley JA, Gardner MA, Stafford EG, Mau TK, Pohlner PG, Tam RK, et al. Late complications of tissue glues in aortic surgery. Ann Thorac Surg. 2000;69:1764-8.

4. Kazui T, Washiyama N, Bashar AH, Terada H, Suzuki K, Yamashita K, et al. Role of biologic glue repair of proximal aortic dissection in the development of early and midterm redissection of the aortic root. Ann Thorac Surg. 2001;72:509-14. 